\title{
Children have lower SARS-CoV-2 viral loads than adults
}

David Curtis

UCL Genetics Institute, UCL, Darwin Building, Gower Street, London WC1E 6BT.

Centre for Psychiatry, Queen Mary University of London, Charterhouse Square, London EC1M 6BQ.

Correspondence:

David Curtis $\underline{\text { d.curtis@ucl.ac.uk }}$ 


\section{Abstract}

Background The question of whether children are less likely to pass on SARS-CoV-2 is important for planning society's response to the pandemic. A document available on the Charité hospital website has not been formally published but is frequently referred to as supporting the notion that viral loads are similar in children and adults. Methods The summary data from this document was extracted and used to produce summary statistics for three age groups: younger children (ages up to 11), older children (ages 12 to 19) and adults (ages 20 and above). Viral loads between the two children's groups and the adult group were compared using Welch's t test, which only requires group means and standard deviations and which is robust against moderate departures from normality. Findings Viral loads were significantly lower than in adults for both younger children $(p=0.04)$ and older children $(p=0.04)$. Overall, viral loads were lower in children than adults $(p=0.005)$. Interpretation For both younger and older children, on average those with detectable SARS-CoV-2 have significantly lower viral loads than adults. Funding No funding was received for this analysis.

\section{Research in context}

\section{Evidence before this study}

Children generally have a less severe response to infection with SARS-CoV-2 and there are as yet unpublished reports that they are also less likely to transmit infection. However one unpublished report from a clinical testing laboratory is widely claimed to show that viral loads in children and adults are similar.

\section{Added value of this study}


This study performs appropriate statistical analyses on the summary results from the laboratory and demonstrates that, contrary to previous claims, children testing positive on average have lower viral loads than adults.

\section{Implications of all available evidence}

The extent to which children do or do not transmit infection should be obtained from high quality, peer-reviewed studies published in reputable scientific journals.

\section{Introduction}

An important issue to be considered for society's response to the SARS-CoV-2 pandemic is the extent to which children pass on infection and this is especially relevant to considerations around schooling arrangements (1). It is clear that children are less susceptible to severe disease and epidemiological studies show lower detectable infection rates (2).

The question of whether children have lower attack rates than adults is hotly debated but studies which formally investigate this have yet to be published in peer reviewed journals. However a report which is frequently referred to in these debates is at time of writing available on the website of Charité - Universitätsmedizin Berlin, a university hospital, at

\section{https://zoonosen.charite.de/fileadmin/user upload/microsites/m cc05/virologie-}

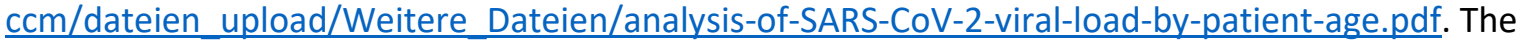
report is titled "An analysis of SARS-CoV-2 viral load by patient age" and appears to be authored by Christian Drosten and colleagues. Although it is written in the format of a scientific paper its status is unclear and it does not seem to have been posted to a preprint server. Notably, it reports on test results obtained from a clinical service but there is no mention of ethical approval for research use. Nevertheless, the document has been widely read and is frequently referred to as showing that children have similar viral loads to adults. A typical usage is in an article appearing in the UK 
newspaper The Guardian on $17^{\text {th }}$ May 2020 titled Scientists divided over coronavirus risk to children if schools reopen which contains the following passage:

However, research carried out by a team led by German coronavirus expert Christian Drosten at the Charité hospital in Berlin found no significant difference between any age categories, including children. "Viral loads in the very young do not differ significantly from those of adults. Based on these results, we have to caution against an unlimited re-opening of schools and kindergartens in the present situation. Children may be as infectious as adults."

In fact, although the Guardian title refers to scientists being "divided", all other scientists quoted broadly agree that children do present less of an infection risk than adults, so the contribution from the Drosten group is the single dissenting view.

There are clearly grounds for concern that the debate about important public health measures is so impacted by a study which has not undergone peer review. However an additional issue is that a superficial view of the document with the results it presents gives the strong impression that children do indeed have lower viral loads than adults, directly contradicting the conclusions quoted above. The document reports rates of positive testing and estimated viral concentrations based on RT-PCR in samples submitted to the Charité Institute of Virology laboratory. Results are expressed as the $\log 10$ of estimated number of viral particles per $\mathrm{ml}$ and broken down by age into either ten groups by decade or six groups by social strata. The results are also displayed in scatter plots which are sometimes copied by commentators to support the claim that viral loads in adults and children are similar. The authors comment that the distribution of viral load values is skewed and that there is a sharp drop on the left side of the distribution because of the assay sensitivity limit.

Here we perform formal statistical analyses to test whether or not children have lower viral loads than adults.

\section{Methods}


Although the document is not formally published it is at time of writing freely available and has been widely circulated and quoted. Hence we regard the summary results reported within it to be in the public domain.

Summary results were obtained from Tables 1 and 2 of the report from the Drosten group. This consisted of the numbers tested, numbers testing positive, and the mean and standard deviation of the estimated viral load by age groups. The results had been summarised over six different age groups, designated as follows: "kindergarten" (ages 0-6); "grade school” (ages 7-11); "high school” (ages 12-19); "university" (ages 20-25); "adult" (26-45 years); "mature" (age over 45). These summary results are displayed in Table 1. In order to investigate the question of whether children tended to have different viral loads from adults, results from these six groups were amalgamated into three groups of interest, consisting of younger children (ages up to 11), older children (ages 12 to 19 ) and adults (ages 20 and above). If we use $\mathrm{N}$ as the number in a group, $\mathrm{m}$ as the mean and $\mathrm{s}$ as the standard deviation then the sum of values in a group is given by $N^{*} \mathrm{~m}$ and the sum of squares of values is given by $(\mathrm{N}-1) *\left(\mathrm{~s}^{2}+\mathrm{m}^{2 *} \mathrm{~N}^{2}\right)$. These sums can then be added across groups to be combined and then the results can be used to recalculate the means and standard deviations for the amalgamated groups. This method does not make any assumptions about the shape of the distribution of the values but simply relies on the definition of the mean and standard deviation. Hence the amalgamated data can be subjected to formal statistical testing without any need for the original individual data values.

The numbers testing positive in each of the child groups were compared with numbers testing positive in the adult group using chi-squared tests. The viral load values of each of the child groups were separately compared to those of the adult group using Welch's $t$ test, which does not require that the variances of the groups be equal and which is robust against moderate departures from normality (3). Welch's $t$ test depends only on knowing the number of subjects, mean and standard deviation for each sample and hence could be applied to the amalgamated data. As a subsidiary 
analysis, the two child groups were combined into one and the viral loads for this combined group were compared to those of the adult group using Welch's $t$ test.

All analyses were carried out using Microsoft Excel for Microsoft 365 MSO (16.0) 64-bit.

The present analysis of summary, anonymised data which is in the public domain does not require ethical approval.

\section{Results}

The summary results for the three groups of interest are shown in Table 2. The proportions testing positive were smaller than adults for both younger children (chi-squared $=69.1,1 \mathrm{df}, \mathrm{p}<0.0001$ ) and older children (chi-squared $=15.5,1 \mathrm{df}, \mathrm{p}<0.001$ ). The mean viral loads with $95 \%$ confidence limits are shown in Figure 1. It can be seen that the mean viral load values for the child groups are lower than for the adult group but that because their sample sizes are relatively small they have wide confidence intervals and these just overlap with the confidence interval for the adult group. On formal statistical testing, the viral load values for children up to age 11 were lower than for adults (mean difference $=0.54$, Welch's $t=2.02, p=0.04$ ) and the viral load values for children between 12 and 19 years were lower than for adults (mean difference $=0.43$, Welch's $t=2.04, p=0.04$ ). In the analysis of all children combined against adults there was clear evidence that they had lower average viral load values (mean difference $=0.47$, Welch's $t=2.78, p=0.005$ )

\section{Discussion}

In the data presented the viral loads, expressed as the logarithm of estimated concentration, are lower in children than adults. The separate tests for younger and older children each yield a $p$ value of 0.04 . If only one had been significant at this level then one would argue that, because of the need to apply a Bonferroni correction for multiple testing, overall the results were not significant. However the probability for both tests to be significant at 0.04 or less is 0.0016 and the test for all children combined is significant at $p=0.005$ so there is little doubt that this is a real difference. 
Given the measurements on the log scale, the best estimate of the magnitude of this difference is that it represents an approximately threefold difference in measured concentrations between children and adults with detectable viral RNA. However one should bear in mind that some subjects may have had viral concentrations below the detectable level and one can note that a smaller proportion of children had detectable virus than adults.

The main reason that the original document reports that there were no significant differences between age groups seems to be that the analyses were based on all pairwise comparisons between all age groups. This weakens the power to test the hypotheses of interest in two different ways. Firstly, it produces reduced numbers in each group. Secondly, it results in multiple comparisons which, if subjected to a Bonferroni correction, yield a threshold for statistical significance which is difficult for any single test to achieve. Using ten age groups results in 45 pairwise comparisons and even using only six groups yields 15 comparisons, meaning that no individual test would be reported to be significant unless it yielded an uncorrected $p$ value less than $0.05 / 15=0.003$.

To conclude, the reported results of testing at the Charité Institute of Virology laboratory show that, in both younger and older children, on average those with detectable virus have lower SARS-CoV-2 viral loads than adults.

\section{References}

1. Munro APS, Faust SN. Children are not COVID-19 super spreaders: time to go back to school. Arch Dis Child [Internet]. 2020 May 5 [cited 2020 May 18]; Available from: http://www.ncbi.nlm.nih.gov/pubmed/32371442

2. Gudbjartsson DF, Helgason A, Jonsson H, Magnusson OT, Melsted P, Norddahl GL, et al. Spread of SARS-CoV-2 in the Icelandic Population. N Engl J Med. 2020 Apr 14;

3. Welch B. The Generalisation of Student's Problems When Several Different Population Variances Are Involved. Biometrika. 1947;34:28-35. 


\section{Table 1}

Summary results obtained from original document on Charité hospital website showing numbers tested for SARS-CoV-2, numbers testing positive and mean and SD of viral load, expressed as the $\log 10$ of estimated particles per $\mathrm{ml}$.

\begin{tabular}{|l|l|r|r|r|r|}
\hline Age & Designation & Count & Positive & $\begin{array}{l}\text { Viral } \\
\text { load }\end{array}$ & \multicolumn{1}{l|}{ SD } \\
\hline $0-6$ & Kindergarten & 1,759 & 37 & 4.371 & 1.601 \\
\hline $7-11$ & Grade school & 623 & 16 & 5.365 & 2.215 \\
\hline $12-19$ & High school & 1,790 & 74 & 4.784 & 1.776 \\
\hline $20-25$ & University & 4,587 & 267 & 5.284 & 1.946 \\
\hline $26-45$ & Adult & 23,665 & 1,247 & 5.159 & 1.971 \\
\hline Over 45 & Mature & 27,407 & 2,071 & 5.229 & 1.867 \\
\hline
\end{tabular}




\section{Table 2}

Results for three amalgamated age groups showing numbers tested SARS-CoV-2, numbers testing positive and mean, SD, SE and 95\% confidence limits for viral load, expressed as the log10 of estimated particles per $\mathrm{ml}$.

\begin{tabular}{|l|l|r|r|l|l|l|l|l|l|}
\hline Age & Designation & Count & Positive & $\begin{array}{l}\% \\
\text { Positive }\end{array}$ & $\begin{array}{l}\text { Viral } \\
\text { load }\end{array}$ & SD & SE & $\begin{array}{l}\text { Low } \\
\text { CL }\end{array}$ & $\begin{array}{l}\text { High } \\
\text { CL }\end{array}$ \\
\hline $0-11$ & Younger children & 2,382 & 53 & 2.2 & 4.67 & 1.92 & 0.26 & 4.15 & 5.19 \\
\hline $12-19$ & Older children & 1,790 & 74 & 4.1 & 4.78 & 1.78 & 0.21 & 4.38 & 5.19 \\
\hline Over 19 & Adults & 55,659 & 3,585 & 6.4 & 5.21 & 1.91 & 0.03 & 5.15 & 5.27 \\
\hline
\end{tabular}




\section{Figure 1}

Mean viral load in each age group expressed as the log10 of estimated particles per $\mathrm{ml}$ along with 95\% confidence intervals.

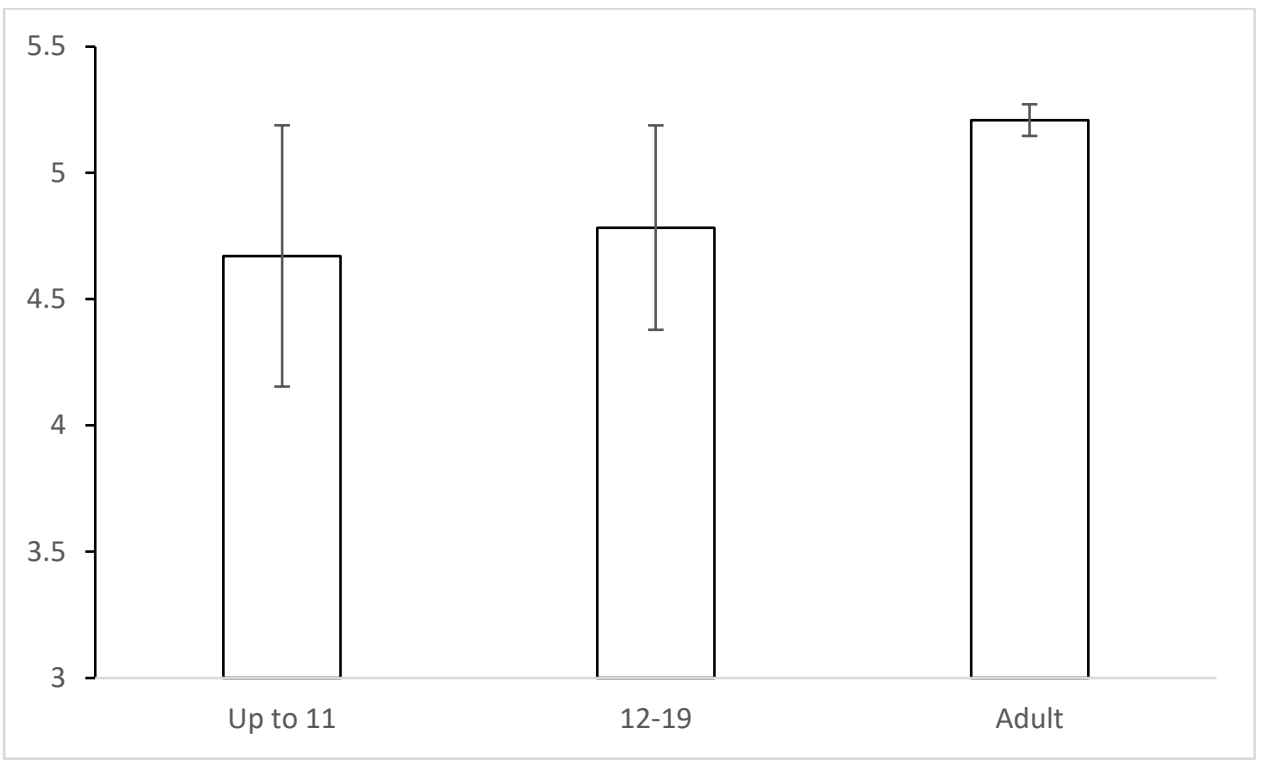

